\title{
Sample storage conditions induce post- collection biases in microbiome profiles
}

\author{
Samir V. Jenkins ${ }^{1}$, Kieng B. Vang ${ }^{2}$, Allen Gies ${ }^{3}$, Robert J. Griffin', Se-Ran Jun, Intawat Nookaew ${ }^{4}$ \\ and Ruud P. M. Dings ${ }^{1 *}$ (i)
}

\begin{abstract}
Background: Here we investigated the influence of different stabilization and storage strategies on the quality and composition of the fecal microbial community. Namely, same-day isolated murine DNA was compared to samples stored for 1 month in air at ambient temperature, with or without preservative buffers (i.e. EDTA and lysis buffer), different temperatures (i.e. $4^{\circ} \mathrm{C},-20^{\circ} \mathrm{C}$, and $-80^{\circ} \mathrm{C}$ ), and hypoxic conditions.

Results: Only storage in lysis buffer significantly reduced DNA content, yet without integrity loss. Storage in EDTA affected alpha diversity the most, which was also reflected in cluster separation. Distinct changes were also seen in the phyla and bacterial species abundance per storage strategy. Metabolic function analysis showed 22 pathways not significantly affected by storage conditions, whereas the tyrosine metabolism pathway was significantly changed in all strategies except by EDTA.
\end{abstract}

Conclusion: Each long-term storage strategy introduced a unique post-collection bias, which is important to take into account when interpreting data.

Keywords: Gut microbiome, 165 rRNA, Alpha diversity, Fecal microbiome transplantations, Metabolic function analysis

\section{Background}

Commensal bacteria reside in many parts of the human body, including the oral cavity, respiratory tract, urogenital tract, skin and maybe most notably the gastro-intestinal tract $[1,2]$. In the human intestine alone, the total number of bacteria is estimated to be around $1 \times 10^{14}(\sim 2 \mathrm{~kg})$, outnumbering the eukaryotic cells by a factor of ten or more $[1,3,4]$. A disturbance in the composition of the microbiota, also termed dysbiosis, may result in an increase in the risk of various diseases, including inflammatory (e.g., inflammatory bowel disease, Crohn's disease, and colon cancer), autoimmune (e.g., celiac disease, arthritis, and multiple sclerosis), allergy-based (e.g., asthma and atopy), metabolic (e.g., diabetes, obesity, metabolic syndrome, and kwashiorkor), and psychological/neurological (e.g., autism) diseases $[1,2,5]$. Moreover, it is becoming increasingly clear that the microbiome is not only integral to providing significant insight into disease states, but it can also be

\footnotetext{
* Correspondence: rpmdings@uams.edu

${ }^{1}$ Department of Radiation Oncology, University of Arkansas for Medical Sciences, Little Rock, AR, USA

Full list of author information is available at the end of the article
}

applied or manipulated in a therapeutic fashion. For example, clinically, fecal microbiome transplantation (FMT) of healthy donors is a successful treatment for Clostridium difficile infection [6].

The most common method to identify commensal bacteria is through next generation sequencing, with $16 \mathrm{~S}$ ribosomal RNA (rRNA) being widely used [7]. 16S sequencing of fecal matter is an effective, non-invasive method to assess the gut microbiome, as it generates significant taxonomic information often to the level of bacterial species. Unfortunately, this sensitivity also means that sample handling can affect the results and introduce a bias within the generated profile.

Thus far, studies have analyzed changes in the bacterial population as a result of different commercially available isolation kits $[8,9]$ or different storage conditions over a relatively short period of time. For example, storage at ambient room temperature $\left(\mathrm{RT} ; \sim 20^{\circ} \mathrm{C}\right)$ for 2 days introduced differences in the population [7, 10]. Similarly, short-term storage in different media, such as lysis buffers or nucleic acid stabilizers (e.g. Ethylenediaminetetraacetic acid (EDTA)) have also been reported to significantly change the microbiome profile $[11,12]$. 
These reports typically use a storage time of one or 2 days as it represents a clinical timescale between a patient providing a sample and the initial analysis. However, basic and translational sciences often rely on longitudinal animal models resulting in multiple samples per individual over time as the disease state progresses. Additionally, in both clinical and academic settings it is often advantageous to work-up many samples at once, rather than immediately upon collection of individual samples. As a result samples are collected and stored for extended periods of time prior to analysis.

Aside from the short-term influences of the various temperatures and stabilization buffers on the microbiome profile, samples are almost always stored under normoxic conditions $\left(\sim 21 \% \mathrm{O}_{2}\right)$. This has the potential, at least in theory, of introducing a false positive bias towards aerobic bacteria during long-term storage.

Here, we investigated the effects of storing fecal samples long-term in a variety of storage conditions (Table 1). The fecal matter of C57BL/6 mice was used, as they are one of the most common animals employed in research, especially in regards to studying gut microbiota related diseases [13]. The bacterial DNA of the same day isolated samples assessed by $16 \mathrm{~S}$ rRNA amplicon sequencing was compared to 1 month of storage at $\mathrm{RT}$, with or without preservative buffers (i.e. $100 \mathrm{mM}$ EDTA and lysis buffer), different temperatures (i.e. $4{ }^{\circ} \mathrm{C}$, $-20^{\circ} \mathrm{C}$, and $-80^{\circ} \mathrm{C}$ ), and hypoxic conditions (i.e. $<10$ $\mathrm{mmHg} \mathrm{pO}_{2}$ ).

\section{Methods}

\section{Fecal collection and storage conditions}

Experiments were approved by the University of Arkansas for Medical Sciences Institutional Animal Care and Use Committee and performed in accordance with relevant regulations and guidelines. Pooled fecal matter of healthy 12 week old female C57BL/6 mice (JAX \#000664, The Jackson Laboratory; $n=5$ ) was collected and apportioned. Individual pellets were collected, pooled, and then apportioned at $200 \mathrm{mg}$ per sample and randomly distributed over the different 8 conditions (Table 1): same day isolation, or stored for 33 days at room temperature (RT), 100 mM EDTA at RT (in $5 \mathrm{ml} 10 \mathrm{mM}$ Tris-HCL pH 8.0), lysis buffer at RT (Invitrogen S1 and S2 solution, \#A29790), refrigerated at $4{ }^{\circ} \mathrm{C}$, frozen at $-20^{\circ} \mathrm{C}$ or $-80^{\circ} \mathrm{C}$ or stored under hypoxic conditions $\left(<10 \mathrm{mmHg} \mathrm{pO}_{2}\right)$ at RT. All samples were stored in the dark, and homogenized immediately prior to DNA extraction.

Hypoxic conditions were created by storing the sample in purged airtight glass syringes (Restek 2.5MDRVLL-GT) in an anaerobe chamber (Forma Scientific Inc.) with a gas mix $\left(5 \% \mathrm{H}_{2}, 5 \% \mathrm{CO}_{2}\right.$ and $\left.90 \% \mathrm{~N}_{2}\right)$ generating hypoxic conditions, as defined as oxygen concen-

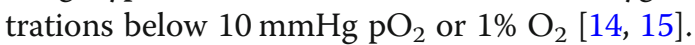

\section{DNA extraction and quantification}

After the long-term storage strategies DNA extraction was performed using PureLink ${ }^{\text {TM }}$ Microbiome DNA Purification Kit (\#A29790; Invitrogen, Carlsbad, CA) according to the manufacturer's instruction. For each storage condition duplicate samples were stored under identical conditions but in separate containers until processing. Briefly, samples were suspended in lysis buffer and heated to $60^{\circ} \mathrm{C}$ for $15 \mathrm{~min}$ prior to $15 \mathrm{~min}$ of horizontal vortexing to homogenize the samples. Samples were centrifuged and the supernatant collected. From this several processing steps were performed to remove residual protein and the final DNA sample was eluted in $100 \mu \mathrm{L}$ of nuclease free $\mathrm{H}_{2} \mathrm{O}$. The concentration and purity were determined by the $\mathrm{A}_{260} / \mathrm{A}_{280}$ value (Cytation 5; BioTek, Winooski VT, USA).

\section{$16 \mathrm{~S}$ rRNA gene sequencing}

The V3 and V4 hypervariable regions of the bacterial $16 \mathrm{~S}$ rRNA gene were amplified using primers containing Illumina adapters following Illumina's 16S Metagenomics sequencing Library Preparation Protocol (\# 15044223, Rev. B) optimized for the Illumina MiSeq system. In brief, Kapa Library Amplification Kit (\# KK2611) was used for polymerase chain reaction (PCR) and products were

Table 1 Sample stabilization and storage conditions

\begin{tabular}{|c|c|c|c|c|c|}
\hline & Classification & Time & Solution & Temperature & Oxygen level $^{a}$ \\
\hline 1. & Fresh & $<1$ days & NA & NA & NA \\
\hline 2. & EDTA & 33 days & 100 mM EDTA & $20^{\circ} \mathrm{C}$ & Ambient \\
\hline 3. & Lysis & 33 days & $\mathrm{S} 1$ and $\mathrm{S} 2^{b}$ & $20^{\circ} \mathrm{C}$ & Ambient \\
\hline 4. & RT & 33 days & NA & $20^{\circ} \mathrm{C}$ & Ambient \\
\hline 5. & $4{ }^{\circ} \mathrm{C}$ & 33 days & NA & $4^{\circ} \mathrm{C}$ & Ambient \\
\hline 6. & $-20^{\circ} \mathrm{C}$ & 33 days & NA & $-20^{\circ} \mathrm{C}$ & Ambient \\
\hline 7. & $-80^{\circ} \mathrm{C}$ & 33 days & NA & $-80^{\circ} \mathrm{C}$ & Ambient \\
\hline 8. & Hypoxia & 33 days & NA & $20^{\circ} \mathrm{C}$ & $<10 \mathrm{mmHg} \mathrm{pO}$ \\
\hline
\end{tabular}

${ }^{\mathrm{a} A m b i e n t} \mathrm{pO} 2$ is anticipated to be $160 \mathrm{mmHg} \mathrm{pO}_{2} \cong 21 \% \mathrm{O}_{2} ; 10 \mathrm{mmHg} \mathrm{pO}_{2} \cong 1.3 \% \mathrm{O}_{2}$

${ }^{\mathrm{b}}$ Proprietary. Obtained from PureLink Microbiome DNA Purification Kit (Invitrogen \#A29790). NA= Not Applicable 
cleaned using Beckman Coulter Agencourt AMPure XP Beads (\# A63881) according to the 16S Metagenomics protocol. The full-length primer sequences used described by Klindworth et al., [16] in standard IUPAC nucleotide nomenclature are:

16S Amplicon PCR Forward Primer $=5^{\prime}$

CG TCG GCA GCG TCA GAT GTG TAT AAG AGA CAG CCT ACG GGA GGC AGC AG

16S Amplicon PCR Reverse Primer $=5^{\prime}$

GTC TCG TGG GCT CGG AGA TGT GTA TAA GAG ACA GGA CTA CCA GGG TAT CTA AT

The resulting products were quantified and $5 \mu$ of each sample was then used for indexing using Illumina's Nextera Dual Indexing Strategy (Additional file 1: Table S1). Subsequently, the indexed samples were cleaned, quantified, and checked for size on an Agilent Tapestation 2200 using D1000 Tapes (\# 5067-5582) and associated reagents (\# 5067-5583). All samples were found to be in the expected size range (600-650 bp) (Additional file 1: Figure S1) and their concentrations were adjusted to $4 \mu \mathrm{M}$ and prepared for loading on the Illumina MiSeq according to Illumina's 16S Metagenomics Protocol. Subsequently they were denatured and loaded on the Illumina MiSeq at $8 \mathrm{pM}$ and sequenced paired-end $(2 \times 300)$ using a MiSeq ${ }^{\circ}$ Reagent Kit v3 (600 cycle) (\# MS-102-3003).

\section{Data processing and analysis}

After demultiplexing the raw reads into individual samples BBmap software was used for preprocessing. Subsequently quality trimming was performed using BBDuk (v.37.02) with a quality score cut-off 15 [17]. The high quality pair-end reads were then merged by BBMerge with an overlap length cut-off region of 22 bases [17]. Reads shorter than $200 \mathrm{bp}$ were discarded and were used in OTU picking step. The OTU picking was performed by QIIME software (v.1.9.1) with open OTU picking workflow using the default option [18]. The results from OTU picking step were imported to the $\mathrm{R}$ suite environment through PhyloSeq packages for statistical analysis and illustration of results [19]. The metabolic capability projection from the detected OTUs was derived from functional profiles inferred by the PanFP method, which can be applied to any OTU picking protocols [20]. The two-tailed Student's t-test and the non-parametric Mann-Whitney were used to determine the validity of differences between the data sets, presented as the mean \pm standard errors of the mean. A $P$ value of 0.05 or less was considered significant.

\section{Results}

Long-term storage in lysis buffer reduces recoverable DNA, without loss of DNA integrity

For each storage condition the DNA isolation efficiency was compared to the yield from samples immediately isolated (Fig. 1). We found based on the absorbance at
$260 \mathrm{~nm}$ that storing the samples in lysis buffer resulted in the lowest nucleic acid yield: in the fresh samples we recovered $95 \pm 25 \mathrm{ng} / \mu \mathrm{l}$ nucleic acid, whereas storage in the lysis buffer reduced this to $16.9 \pm 1.5 \mathrm{ng} / \mu \mathrm{l}$ (Fig. 1a). None of the other storage conditions caused a significant drop in nucleic acid yield. These trends were also seen in the double stranded DNA (dsDNA) yields. Whereas fresh samples resulted in an average of $60.4 \pm 18.6 \mathrm{ng} / \mu \mathrm{l}$ dsDNA, storage in lysis buffer reduced this to $12.7 \pm 0.6 \mathrm{ng} / \mu \mathrm{l}$ (Fig. $1 \mathrm{~b}$ ). Although the lysis buffer reduced both the nucleic acid and the dsDNA content, the integrity was maintained. Namely based on the Taqman analysis (Additional file 1: Figure S1) and the total reads, storing the sample in lysis buffer did not interfere with the quality of the bacterial DNA. Storing at RT $\left(\sim 20^{\circ} \mathrm{C}\right)$, however, did reduce the integrity. Whereas fresh isolations rendered on average $8.8 \times 10^{5}$ total reads, storing the sample at RT provided only $5.6 \times 10^{5}$ total reads on average, a reduction of almost $40 \%(p<0.05$; Fig. 1c). The other storage conditions did not alter the number of reads significantly.

\section{Sample storage in EDTA reduces the alpha diversity}

In order to assess whether storage condition could alter the alpha diversity, the variety within a sample, we quantified the amount of observed operational taxonomic units (OTUs) richness (Fig. 2). Of the different storage conditions, lysis buffer caused a modest drop in observed OTUs from 2205 OTUs to 1750 OTUs as compared to immediate isolations of fresh samples. Long-term storage of the samples in EDTA, however, caused a significant drop in OTUs down to 1321 OTUs $(p<0.05)$. Keeping the samples for an extended time at different temperatures did not significantly change the observed OTUs, nor did storing the under hypoxic conditions. We also analyzed the alpha diversity by the Chao1 estimator and found the same trends as seen for the observed OTUs (Additional file 1: Figure S2).

\section{Microbiota ordination and taxon stored in lysis buffer clusters with fresh isolated samples}

Principal coordinate analysis (PCoA) of Bray Curtis distances revealed that the different storage conditions caused 3 clusters (Fig. 3). Notably the fresh isolated bacterial DNA clustered best with the DNA isolated from samples stored with the lysis buffer. The samples stored at different temperature ranging from $-80^{\circ} \mathrm{C}$ to $20^{\circ} \mathrm{C}$ also clustered together. Anaerobic stored samples clustered by themselves and samples stored in EDTA changed the most and were separated from the other clusters (Fig. 3). 

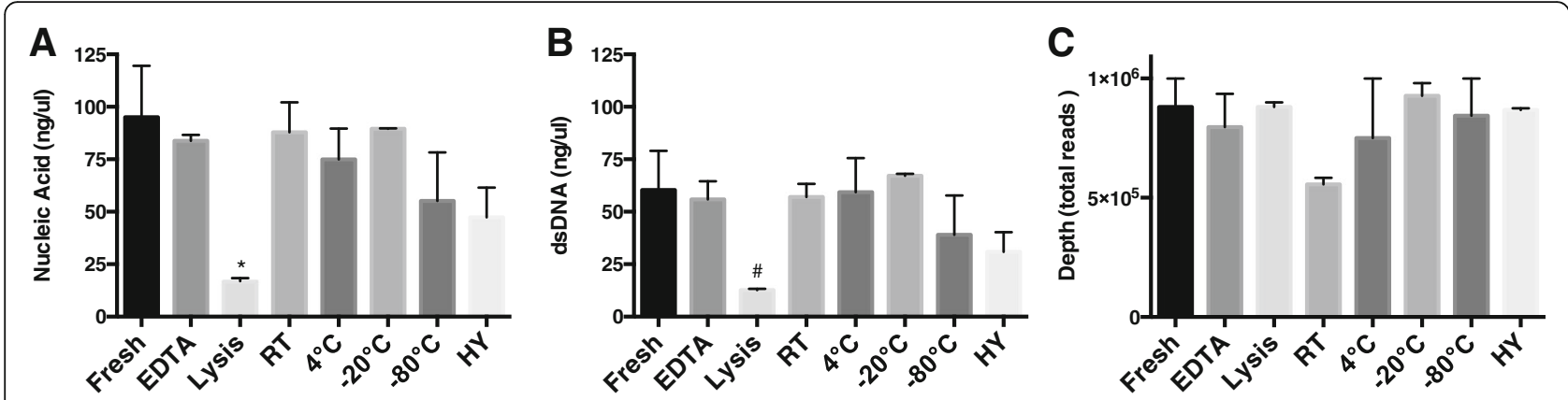

Fig. 1 Recoverable DNA from fecal matter under 8 different storage conditions. a. Total nucleic acid content recovered from fecal matter based on the absorbance at $260 \mathrm{~nm}$. b. Double stranded DNA (dsDNA) concentrations recovered from fecal matter based on the fluorescent absorbance of the Qubit dsDNA broad range (BR) assay reagent. c. Bacterial DNA sequencing coverage achieved per different storage condition. The data is presented as the mean \pm SEM. ${ }^{*} p<0.05,{ }^{*} p=0.06$

\section{Hypoxic storage conditions induces the greatest} significant changes in relative Phyla abundance

Relative phyla abundance analysis identified Firmicutes (68\%) and Bacteroidetes (22\%) as the two dominant phyla in the freshly isolated samples (Table 2 and Fig. 4). This relative abundance of the Firmicutes was reduced by all storage conditions to various degrees, with the greatest relative reduction of $-27.5 \%$ by hypoxic conditions $(p<0.05)$. Universally this relative abundance reduction in Firmicutes was associated with an increase in Bacteroidetes with the greatest increase while stored under hypoxic conditions $(+32.7 \% ; p<0.05)$. Whereas all storage conditions reduced the relative abundance of Actinobacteria, storage in lysis buffer caused a doubling of relative abundance from 6 to $12 \%(p<0.05)$. Overall, as compared to freshly isolated samples, storage under hypoxic conditions was the only strategy that caused significant changes in the relative abundance of all phyla (Table 2 and Fig. 4).

\section{Metabolic function analysis}

The pangenome-based functional profiles (PanFP) method was used to infer functional profiles and the changes induced by the storage conditions. The analysis was performed based on 5110 genes grouped in 276 associated pathways (Table 3 and Additional file 1). Every individual long-term storage condition had some degree of modulation on a genomic as well as pathway level as compared to fresh samples. On a genomic level, storing under hypoxic conditions or at $4{ }^{\circ} \mathrm{C}$ induced the most significant changes in relative gene expression $(\sim 65-$ $69 \%$, Table 3). Storing at $-20^{\circ} \mathrm{C}$ caused significant changes in approximately $52 \%$ of the genes, whereas storing the samples in lysis buffer, EDTA, RT, or $-80^{\circ} \mathrm{C}$

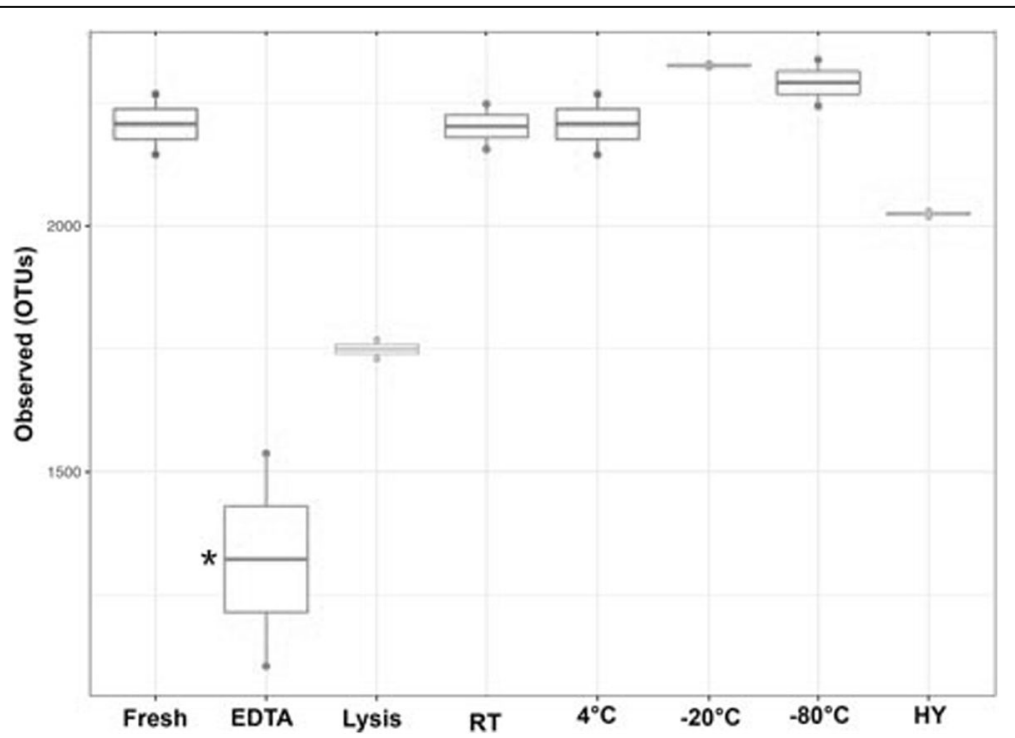

Fig. 2 The observed unique operational taxonomic units (OTUs) between storage conditions and individual samples. The boxplots show the alpha diversity as median, quartile, smallest and largest observations (circles). ${ }^{*} p<0.05$ 


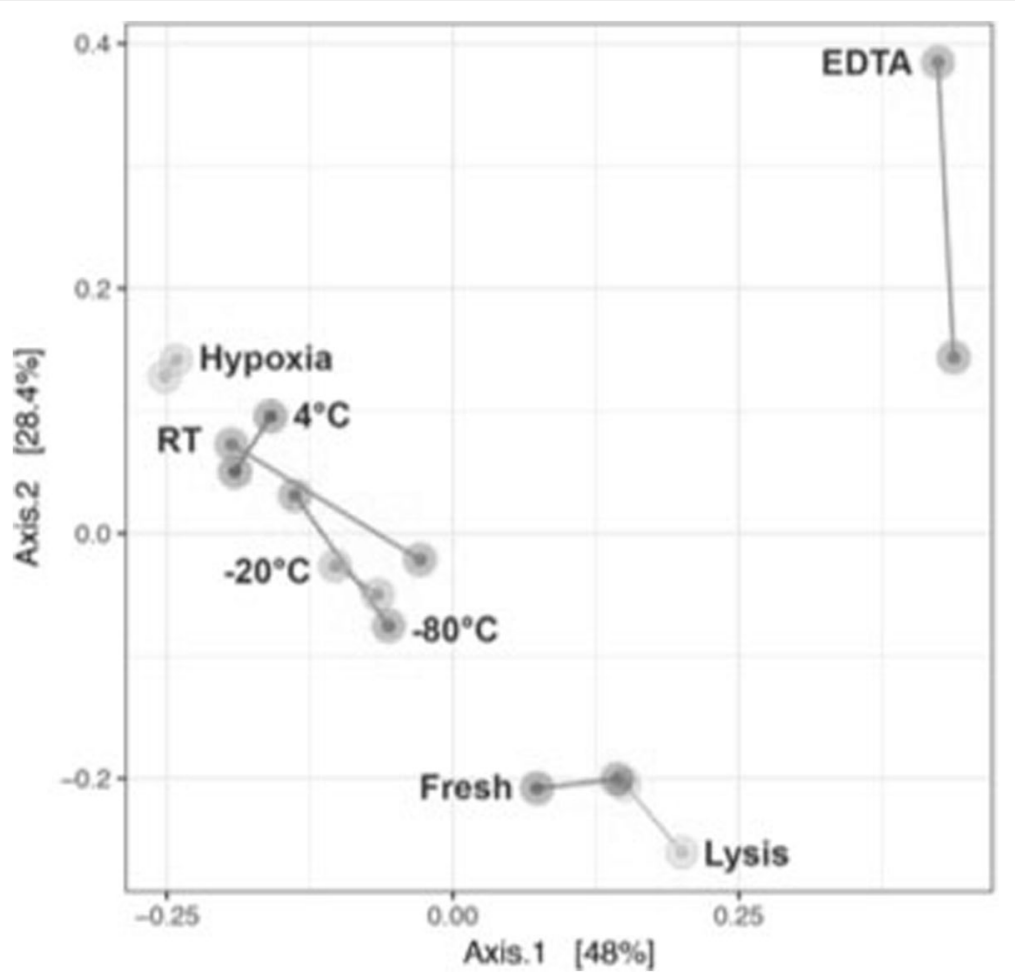

Fig. 3 Clustering of samples by principal coordinate analysis (PCOA). PCoA based on Bray-Curtis dissimilarity. The first two principle coordinates are plotted on the $x$ - and $y$-axis, respectively. This represents $76.4 \%$ of the total variation

caused the fewest significant changes in relative gene expression namely $\sim 14-21 \%$. Pathway analysis showed similar trends. Long-term storage under hypoxic condition, or at $4{ }^{\circ} \mathrm{C}$ and $-20^{\circ} \mathrm{C}$ caused the most significant changes $(\sim 64-72 \%)$; EDTA, lysis buffer, RT and $-80^{\circ} \mathrm{C}$ cause fewer changes $(\sim 10-18 \%)$. None of the pathways were affected by all of the storage strategies. However, the tyrosine metabolism pathway (KEGG pathway ko00350) was most often affected by different conditions: 6 out of the 7 storage strategies affected this pathway significantly. Storage in EDTA, however, did not increase or suppress its relative expression as compared to immediately isolated. Nearly $8 \%$ (22 pathways) were not significantly affected by any long-term storage strategy (Table 3 and Additional file 1). Further analysis revealed that some storage conditions saw enhancement of pathways as their greatest relative change, i.e. apoptosis (ko04214) for $4{ }^{\circ} \mathrm{C}$ storage $(p<0.00001)$, fatty acid biosynthesis $($ ko00061) for hypoxic conditions $(p<0.0003)$ and toluene degradation (ko00623) for EDTA storage $(p<0.0003)$ and for lysis buffer $(p<0.0006)$.

The other storage conditions induced a suppression of pathways as their greatest relative changes, i.e. Alzheimer disease $(k o 05010)$ for $-20^{\circ} \mathrm{C}(p<0.0006)$, lysine degradation (ko00310) for RT $(p<0.003)$ and beta-lactam resistance (ko01501) for $-80^{\circ} \mathrm{C}(p<0.005)$.

\section{Discussion}

Sequencing-based assessment of the fecal microbiome has become increasingly important in science and clinical practice as more correlations and causal relationships are being identified between disease states and microbiome profiles. Therefore it is crucial that no post-collection bias is introduced during long-term sample storage as this might mask proper clinical diagnosis. Here we investigated the influence of different stabilization and storage strategies, i.e. different preservative buffers, temperatures, and oxygen concentrations, on the quality and composition of murine fecal microbiome after being stored for over 1 month.

Interestingly, we found that storage in commercially available lysis buffer reduced the DNA content the most, yet did not cause loss of DNA integrity. Although DNA binding to the plastic microfuge tubes could be a possible explanation, this is unlikely as all samples were stored in the commercially provided receptacles and moreover homogenization by bead-beating was performed just prior to the DNA isolation. It is more likely a function of the storage in a liquid medium causing non-specific DNA degradation. However, this degradation was not observed with the samples stored in EDTA, as EDTA inhibits DNA degradation. All other storage conditions did not induce significant changes in DNA content or integrity. It must be noted that the 


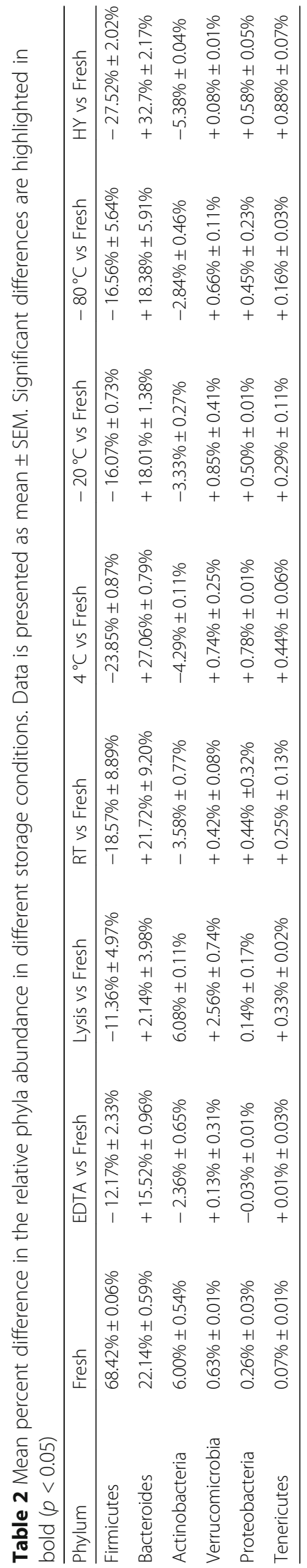



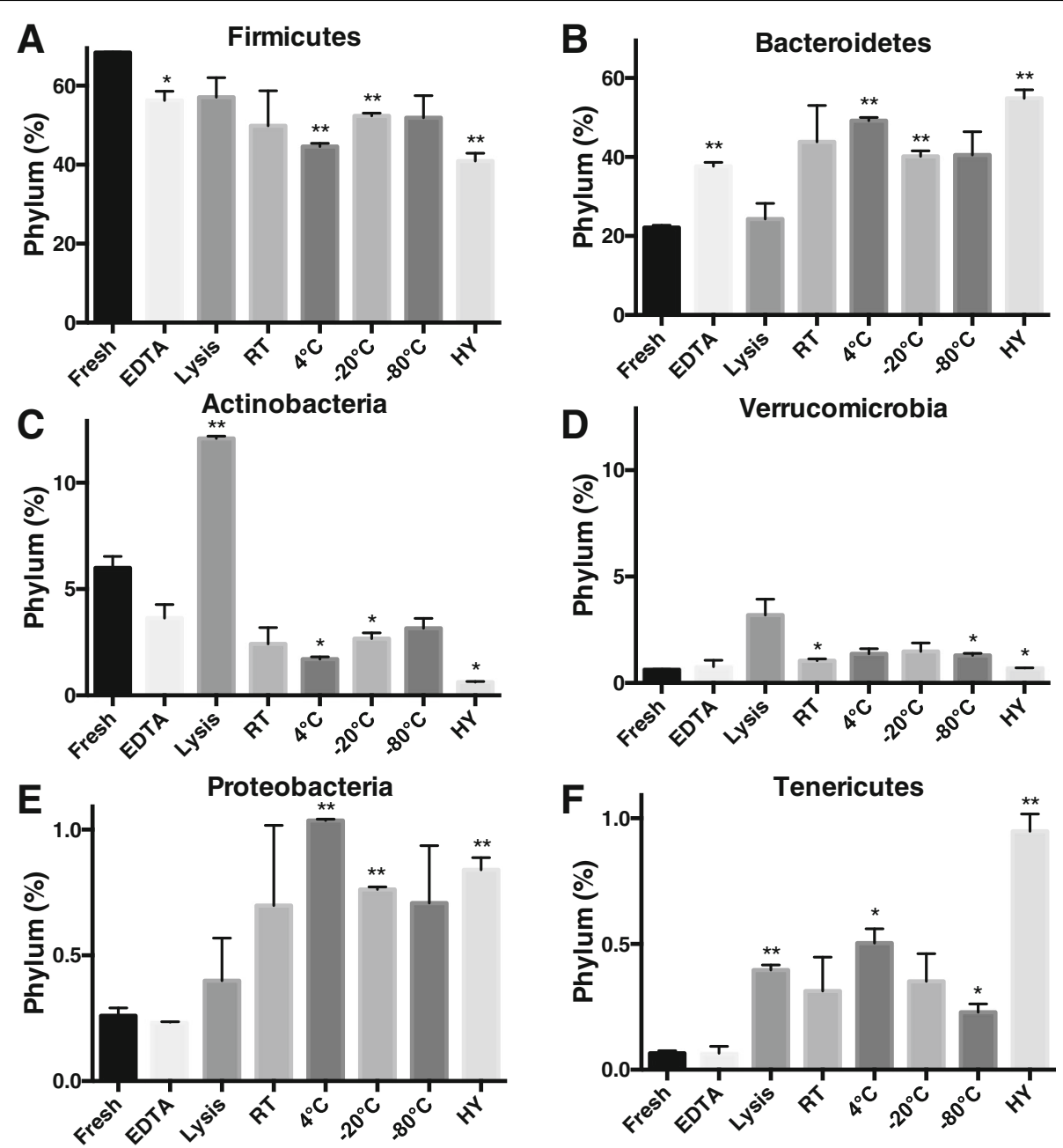

Fig. 4 Phylum composition diversity following incubation under 8 different storage conditions. The relative abundance of (a) Firmicutes, (b) Bacteroidetes, (c) Actinobacteria, (d) Verrucomicrobia, (e) Proteobacteria and (d) Tenericutes as a function of storage condition. ${ }^{*} p<0.05,{ }^{* *} p<0.01$. The data is presented as the mean \pm SEM

Table 3 Functional metabolic genes and pathways affected per storage condition as compared to fresh samples

\begin{tabular}{|c|c|c|c|c|c|c|c|}
\hline \multirow[t]{2}{*}{ Condition } & \multicolumn{2}{|l|}{ Genes $^{a}$} & \multicolumn{5}{|c|}{ Pathways $^{b}$} \\
\hline & Number & $\%$ & Number & $\%$ & Greatest change & $\log 2 \mathrm{FC}$ & $p$ value \\
\hline Fresh & 5110 & 100 & 276 & 100 & NA & NA & NA \\
\hline EDTA & 978 & 19.1 & 27 & 9.8 & Toluene degradation & +3.9 & $<0.0003$ \\
\hline Lysis & 689 & 13.5 & 40 & 14.5 & Toluene degradation & +4.5 & $<0.0006$ \\
\hline RT & 1050 & 20.5 & 40 & 14.5 & Lysine degradation & -2.1 & $<0.003$ \\
\hline $4^{\circ} \mathrm{C}$ & 3533 & 69.1 & 198 & 71.7 & Apoptosis & +3.2 & $<0.00001$ \\
\hline$-20^{\circ} \mathrm{C}$ & 2637 & 51.6 & 183 & 66.3 & Alzheimer Disease & -1.9 & $<0.0006$ \\
\hline$-80^{\circ} \mathrm{C}$ & 1088 & 21.3 & 50 & 18.1 & Beta-lactam resistance & -2.0 & $<0.005$ \\
\hline HY & 3317 & 64.9 & 176 & 63.8 & Fatty acid biosynthesis & +5.3 & $<0.0003$ \\
\hline \multicolumn{3}{|c|}{ Pathways not affected } & 22 & 8.0 & NA & NA & NA \\
\hline \multicolumn{3}{|c|}{ Affected by all conditions } & 0 & 0 & none & NA & NA \\
\hline \multicolumn{3}{|c|}{ Pathway affected by 6 out of 7 conditions (excludes EDTA) } & 1 & 0.4 & Tyrosine metabolism & varies & varies \\
\hline
\end{tabular}


proprietary lysis buffer was not optimized for long-term storage purposes. Many studies have historically stored their samples in EDTA to inhibit the enzymatic action of DNase [11]. We found that long-term storage in EDTA affected the alpha diversity the most, which was also reflected in cluster separation as determined by PCoA.

The overall bacterial taxonomic groups found in murine fecal matter were similar to previous findings, with Firmicutes and Bacteroidetes being the two major phyla [21-23].

Until now this difference has been mostly attributed to differences in species, mouse strains, mouse vendors, or diet [23]. Here we uncovered a new potential confounder, namely storage condition, as this can also significantly influence the measured abundance and composition of the microbiome. We found that the dominance of Firmicutes over Bacteroidetes is minimized over long-term storage or even reversed when stored at $4{ }^{\circ} \mathrm{C}$ or under hypoxic conditions. It is unclear whether this is due to the relative enhanced DNA degradation of the Bacteriodetes or because of a growth-related increase of Firmicutes. This post-collection augmentation is crucial to take into account while interpreting data and moreover regarding fecal microbiome transplantations (FMT), which have been increasingly adapted into clinical practice [6].

On the other hand, this change can also be used to one's advantage when certain phyla or bacterial strains are preferred. For instance, in the past Escherichia coli was falsely interpreted as the primary gut microbe due to the ease with which it could be cultured and detected [24]. Extended storage in relatively oxygen rich conditions favored the growth of (facultative) aerobes, which ultimately affected the measured population distribution and misrepresented the importance and significance of anaerobes. Here we found that storage under hypoxic conditions favors bacterial strains within the Bacteroidetes and Proteobacteria phyla as their relative abundance increased without significant loss in DNA content or integrity. Thus one might consider long-term storage under low oxygen tension when there is an interest in an in-depth analysis of facultative or obligate anaerobe bacteria commonly found within Bacteroidetes and Proteobacteria phyla. Hypoxic conditions also induced changes in regard to metabolic function on a genomic as well as pathway level. The greatest change was observed within the enhancement of the fatty acid biosynthesis pathway- an anaerobic pathway for synthesizing unsaturated fatty acids, possibly due to the increase in Tenericutes [25].

PanFP, a metagenome inference software, allows for functional profiling of the $16 \mathrm{~S}$-based microbiome, generating a rudimentary prediction of the metabolic capacity of the microbiome. Here, we examined whether there were any general changes regarding the metabolic capacity of the microbiome in response to storage conditions. PanFP analysis indicated that the common method of storage at $-80^{\circ} \mathrm{C}$ caused a relative change in more than $21 \%$ of the genes and approximately $18 \%$ of the associated pathways, as compared to the immediately isolated samples. Storage in preservation and stabilization buffers induced fewer significant changes as compared to immediate isolation of fresh samples. However these conditions came at the cost of a significant reduction in DNA content for lysis buffer or a reduction in OTUs, cluster separation and significant changes in phylum distribution for EDTA. Intriguingly, storage at $20^{\circ} \mathrm{C}$ had more than twice the genomic changes and more than thrice the metabolic pathways changes as compared to $-80^{\circ} \mathrm{C}$ (Table 3). Moreover, long-term storage at $4{ }^{\circ} \mathrm{C}$ caused the most modulations, on both the genomic as well as pathway level, in almost two-third of the cases. Thus taken together the coldest condition, $-80^{\circ} \mathrm{C}$, is preferred when immediate isolation is not possible or practical.

From a pathway perspective $8 \%$ of the pathways were not affected by any of the investigated storage conditions. However the genes associated with the tyrosine metabolism pathway were affected in 6 out of 7 conditions. Thus tyrosine metabolism investigations would be preferred on fresh samples only (Table 3). Although PanFP was developed and optimized for $16 \mathrm{~S}$ amplicon sequence data [20], further profiling by e.g. whole-genome shotgun metagenomic sequencing would strengthen these predictions.

\section{Conclusions}

All 7 different strategies introduced a unique post-collection bias. Thus it is important to take this into account during the data interpretation of past, current, or future microbiome profile studies, as well as during therapeutic approaches involving stool-derived treatments (e.g. FMT). Alternatively, this post-collection bias can be used to an advantage if one favors a particular sub-set of phyla or bacteria (e.g. anaerobes), which can be relatively enriched during long-term storage.

\section{Additional file}

Additional file 1: Table S1. The indexing scheme. Datasheet metabolic function gene and pathway analysis. Figure S1. Tapestation 2200 images using D1000 Tapes and D1000 reagents. a, Indexed samples were found to be in the expected size range $(600-650 \mathrm{bp})$. B, Sizes corresponding to the bands detected in the ladder in the first lane of panel A. Figure S2. Detected alpha diversity in fecal matter following incubation under 8 different storage conditions. Chao1 richness estimator between storage conditions and individual samples. (DOCX 438 kb)

\section{Abbreviations}

dsDNA: Double stranded DNA; EDTA: Ethylenediaminetetraacetic acid; FMT: Fecal microbiome transplantation; HY: Hypoxia; OTU: Operational taxonomic units; PCoA: Principal coordinate analysis; RT: Room temperature 


\section{Acknowledgements}

Not applicable.

\section{Funding}

This work was supported by a grant from the Arkansas Biosciences Institute and the Winthrop P. Rockefeller Cancer Institute (RPMD). The study was also supported in part by the Center for Microbial Pathogenesis and Host Inflammatory Responses grant P20GM103625 (RD) and P20GM125503 (IN) through the NIH National Institute of General Medical Sciences Centers of Biomedical Research Excellence. The funders had no role in study design, data collection and analysis, decision to publish, or preparation of the manuscript.

The content is solely the responsibility of the authors and does not necessarily represent the official views of the NIH or UAMS/WPRCI.

\section{Availability of data and materials}

Supplementary data related to this article includes: Indexing scheme, Tapestation 2200 images, Chao1 estimator (PDF) are found in Additional file 1. The datasheet of metabolic function gene and pathway analysis (Excel) is available by reasonable request via the corresponding author.

\section{Authors' contributions}

Design of the work (SJ, RD); data collection (AG); data analysis and interpretation (SJ, KV, AG, RG, IN, SJ, RD); drafting the article (RD); Critical revision of the article (SJ, KV, AG, RG, SJ, IN, RD). All authors have read and approved the manuscript.

\section{Competing interest}

The authors declare that they have no competing interest.

\section{Ethics approval and consent to participate}

No human subjects or their materials were in this study. The animal studies were approved by the University of Arkansas for Medical Sciences Institutional Animal Care and Use Committee (Protocol \#3610) and performed in accordance with relevant regulations and guidelines.

\section{Consent for publication}

Not applicable.

\section{Publisher's Note}

Springer Nature remains neutral with regard to jurisdictional claims in published maps and institutional affiliations.

\section{Author details}

${ }^{1}$ Department of Radiation Oncology, University of Arkansas for Medical Sciences, Little Rock, AR, USA. ${ }^{2}$ Center for Integrative Nanotechnology Sciences, University of Arkansas, Little Rock, AR, USA. ${ }^{3}$ Department of Microbiology and Immunology, University of Arkansas for Medical Sciences, Little Rock, AR, USA. ${ }^{4}$ Department of Biomedical Informatics, University of Arkansas for Medical Sciences, Little Rock, AR, USA

Received: 18 June 2018 Accepted: 28 November 2018

Published online: 27 December 2018

\section{References}

1. Cheng M, Qian L, Shen G, Bian G, Xu T, Xu W, Shen G, Hu S. Microbiota modulate Tumoral immune surveillance in lung through a gammadeltaT17 immune cell-dependent mechanism. Cancer Res. 2014;74:4030-41.

2. Willing BP, Russell SL, Finlay BB. Shifting the balance: antibiotic effects on host-microbiota mutualism. Nat Rev Microbiol. 2011;9(4):233-43.

3. Gagliani N, Hu B, Huber S, Elinav E, Flavell RA. The fire within: microbes inflame tumors. Cell. 2014;157(4):776-83.

4. Ferreira MR, Muls A, Dearnaley DP, Andreyev HJ. Microbiota and radiationinduced bowel toxicity: lessons from inflammatory bowel disease for the radiation oncologist. Lancet Oncol. 2014;15(3):e139-47.

5. Erturk-Hasdemir D, Kasper DL. Resident commensals shaping immunity. Curr Opin Immunol. 2013;25(4):450-5.

6. Khoruts A, Sadowsky MJ. Understanding the mechanisms of faecal microbiota transplantation. Nat Rev Gastroenterol Hepatol. 2016;13(9):508-16.
7. Lane DJ, Pace B, Olsen GJ, Stahl DA, Sogin ML, Pace NR. Rapid determination of $16 \mathrm{~S}$ ribosomal RNA sequences for phylogenetic analyses. Proc Natl Acad Sci U S A. 1985;82(20):6955-9.

8. Song SJ, Amir A, Metcalf JL, Amato KR, Xu ZZ, Humphrey G, Knight R. Preservation methods differ in fecal microbiome stability, affecting suitability for field studies. mSystems. 2016;1(3):1-12.

9. Hart ML, Meyer A, Johnson PJ, Ericsson AC. Comparative evaluation of DNA extraction methods from feces of multiple host species for downstream next-generation sequencing. PLoS One. 2015;10(11):1-16.

10. Dethlefsen $L$, Huse $S$, Sogin ML, Relman DA. The pervasive effects of an antibiotic on the human gut microbiota, as revealed by deep $16 \mathrm{~S}$ rRNA sequencing. PLoS Biol. 2008;6(11):2383-400.

11. Carozzi FM, Sani C. Fecal collection and stabilization methods for improved fecal DNA test for colorectal cancer in a screening setting. J Cancer Res. 2013;2013:1-8.

12. Choo JM, Leong LE, Rogers GB. Sample storage conditions significantly influence faecal microbiome profiles. Sci Rep. 2015:5:1-10.

13. Rabot $S$, Membrez M, Bruneau A, Gerard P, Harach T, Moser M, Raymond F, Mansourian R, Chou CJ. Germ-free C57BL/6J mice are resistant to high-fatdiet-induced insulin resistance and have altered cholesterol metabolism. FASEB J. 2010;24(12):4948-59.

14. Koonce NA, Levy J, Hardee ME, Jamshidi-Parsian A, Vang KB, Sharma S, Raleigh JA, Dings RP, Griffin RJ. Targeting artificial tumor stromal targets for molecular imaging of tumor vascular hypoxia. PLoS One. 2015;10(8):1-12.

15. Griffin RJ, Koonce NA, Dings RP, Siegel E, Moros EG, Brauer-Krisch E, Corry PM. Microbeam radiation therapy alters vascular architecture and tumor oxygenation and is enhanced by a galectin- 1 targeted anti-angiogenic peptide. Radiat Res. 2012;177(6):804-12.

16. Klindworth A, Pruesse E, Schweer T, Peplies J, Quast C, Horn M, Glockner FO. Evaluation of general $16 \mathrm{~S}$ ribosomal RNA gene PCR primers for classical and next-generation sequencing-based diversity studies. Nucleic Acids Res. 2013;41(1):1-11

17. Bushnell B. BBMap short read aligner. Berkeley: University of California; 2016 http://sourceforge.net/projects/bbmap

18. Caporaso JG, Kuczynski J Stombaugh J, Bittinger K, Bushman FD, Costello EK, Fierer N, Pena AG, Goodrich JK, Gordon Jl, et al. QIIME allows analysis of highthroughput community sequencing data. Nat Methods. 2010;7(5):335-6.

19. McMurdie PJ, Holmes S. Phyloseq: an R package for reproducible interactive analysis and graphics of microbiome census data. PLoS One. 2013:8(4):1-11.

20. Jun SR, Robeson MS, Hauser LJ, Schadt CW, Gorin AA. PanFP: pangenome-based functional profiles for microbial communities. BMC Res Notes. 2015:8:479.

21. Ley RE, Hamady M, Lozupone C, Turnbaugh PJ, Ramey RR, Bircher JS, Schlegel ML, Tucker TA, Schrenzel MD, Knight R, et al. Evolution of mammals and their gut microbes. Science. 2008;320(5883):1647-51.

22. Benson AK, Kelly SA, Legge R, Ma F, Low SJ, Kim J, Zhang M, Oh PL, Nehrenberg D, Hua K, et al. Individuality in gut microbiota composition is a complex polygenic trait shaped by multiple environmental and host genetic factors. Proc Natl Acad Sci U S A. 2010;107(44):18933-8.

23. Gu S, Chen D, Zhang JN, Lv X, Wang K, Duan LP, Nie Y, Wu XL. Bacterial community mapping of the mouse gastrointestinal tract. PLoS One. 2013;8(10):1-9.

24. Tenaillon O, Skurnik D, Picard B, Denamur E. The population genetics of commensal Escherichia coli. Nat Rev Microbiol. 2010;8(3):207-17.

25. Zhu L, Cheng J, Luo B, Feng S, Lin J, Wang S, Cronan JE, Wang H. Functions of the Clostridium acetobutylicium FabF and FabZ proteins in unsaturated fatty acid biosynthesis. BMC Microbiol. 2009:9:1-11.

Ready to submit your research? Choose BMC and benefit from

- fast, convenient online submission

- thorough peer review by experienced researchers in your field

- rapid publication on acceptance

- support for research data, including large and complex data types

- gold Open Access which fosters wider collaboration and increased citations

- maximum visibility for your research: over $100 \mathrm{M}$ website views per year

At BMC, research is always in progress.

Learn more biomedcentral.com/submission 